\title{
The Study of Power Grid Planning Based on the Model "One Point and Two Lines, Classification and Subarea”
}

\author{
Lin Qi-you \\ Wuhu Power Supply Company \\ Wuhu, China \\ njhnlqy@sina.com
}

\author{
Wang Ling \\ Qianxinan Nationality Vocational and Technical \\ College \\ Xingyi, China
}

\begin{abstract}
As one of the important sectors of the national economy, the power industry management department should constantly expand the scale of power system. Therefore, it faces increasingly heavy grid development planning task. Based on the research of the vacant area inference, existing classification and subarea method, this paper proposes an improved usage method of classification and subarea. As for existing areas with history load, existing and new areas with no history data, we use different load forecast ideas, which based on the concept of vacant area inference and the current situation of the basic data collection of domestic spatial load forecasting. Meanwhile, according to the classification ideology, taking " Distribution Network Planning and Design of the Technical Guidelines " as guidance, we can establish models in different voltage levels, the grid can be divided into highvoltage distribution network planning model and the medium voltage distribution network planning model. Finally, according to "Distribution Network Planning and Design of the Technical Guidelines", divide the power supply area and propose the grid partition plan model.
\end{abstract}

Keywords- Power system; one point and two lines; classification and subarea; grid planning; time-space load forecasting.

\section{INTRODUCTION}

For a long time, there is a gap between time and space on research efforts and wide scale in power grid planning. Specific performance: network planning often advances in units of time (usually a year), what was given is predicted power in the region year by year and load values, and grid construction projects for the year. The city as a comprehensive development body shows a greater difference in the spatial dimension, but when simply let time dimension as a reference to the network planning, spatial differences between various urban areas will be ignored. Therefore, the time-space network planning put the State Grid' s "Urban Power Network Planning and Design Guidelines " and "Distribution Network Planning and Design of the Technical Guidelines" as the starting point, and explore two main lines of time and space, which are harmony and unified is necessary ${ }^{[1-7]}$.

Classification and subarea is the only way in which grid develops. Classification means dividing network into different voltage levels. Subarea means dividing network into different regions within the same voltage level ${ }^{[8]}$.According to different voltage levels, network can be divide into transmission network (also known as power transmission network), high voltage distribution network and medium voltage distribution network. Different voltage levels grid operate respectively ${ }^{[9-15]}$.

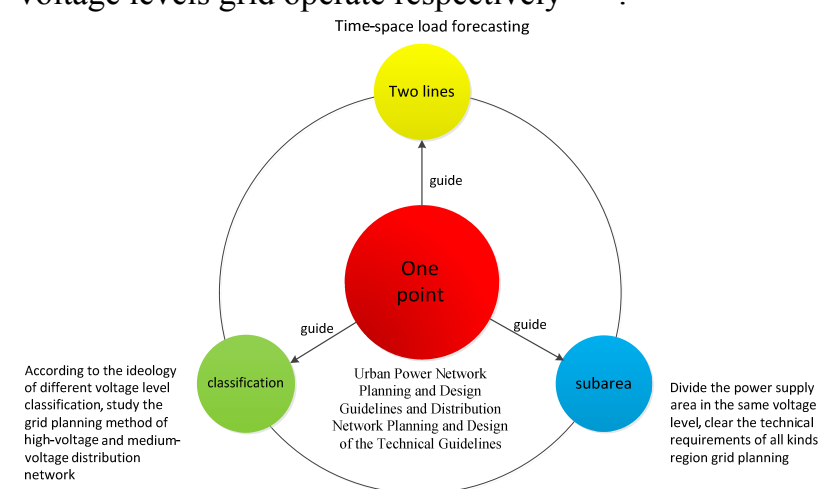

Figure 1. Network planning model proposed

\section{TIME-SPACE LOAD FORECASTING MODEL}

\section{A. Existing areas load distribution forecasting}

"Existing areas" refers to the existing load, whose site features have almost no major change in the urban areas. In practice, the existing areas' historical load data is uneven, and some old town retains a certain historical load data, some historical data are not available. Based on the concept of vacant area inference, doing load distribution forecasting to existing areas which have historical load data and which don't have respectively.

For existing areas with historical load data, according to the actual experience of urban distribution network planning, to gain part of load areas' annual electricity consumption by dividing urban distribution, then we can know load areas' annual maximum load through maximum load utilization hours. For these load areas which have historical load data, we can use regression curve fit load historical value and the saturation value of the vision year, then pushback to get the load of planning period, without calculating by the average density of classification load. The flow of load distribution forecasting of existing areas which have historical data shows below. 


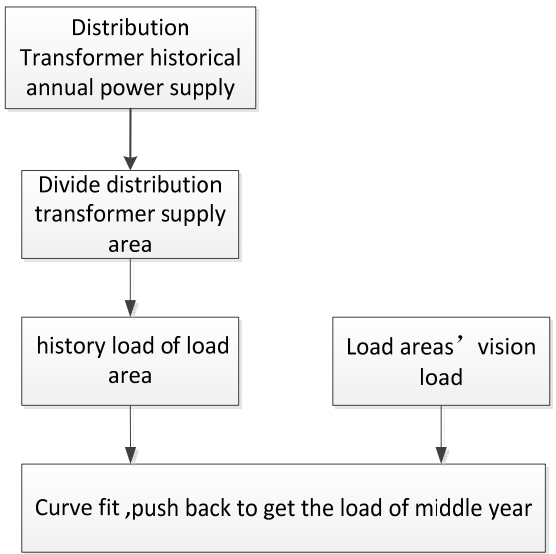

Figure 2.The flow chart of load distribution forecasting of existing areas which have history data

Except above mentioned existing areas, other existing areas' historical load data is unknown, the load distribution can be predicted based on the ideology of their classification load average density.

First, determine the whole existing areas classification load development curve of the status quo, as shown in the following figure. According to the classification of the total area of vision year classification load saturation density and status of the existing areas, calculate vision year classification load of the existing areas. Use mathematical models such as S-shaped curve, the natural logarithm of the polynomial, mixing power polynomial fit the saturation value and historical classification value, and ultimately select the most reasonable development as the classification load curve.

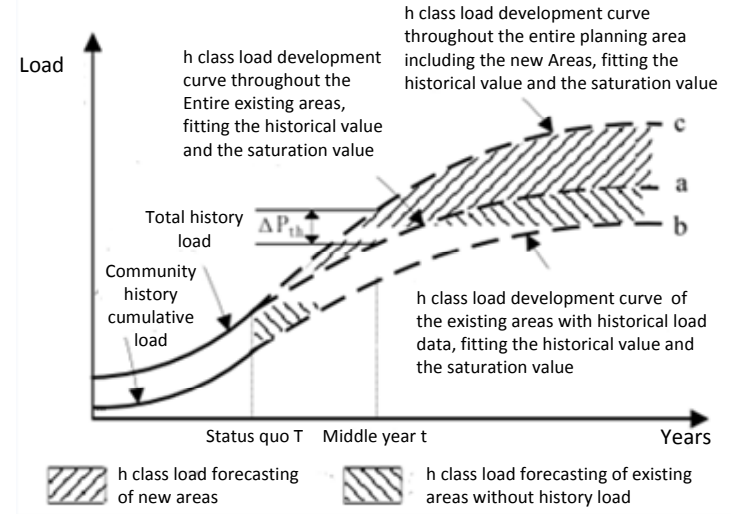

Figure 3. Schematic diagram of existing and new areas $h$ class (h for classification label) load forecasting without history load based on vacant area inference

Then, gather load forecasting values of all existing areas with history data year by year (considering the same time rate), we can get classification load development curve of existing areas with history data, as the curve $b$ shown in the above figure. Finally, the load of similar existing areas with no history data can be calculated by the same classification load average density, the function of the same classification load average density is:

$$
d_{t h 1}^{\prime}=\frac{L_{t h 1}}{S_{1}}=\frac{L_{t h}{ }^{\prime}-L_{t h 2}}{S_{1}}
$$

In the formula, $L_{t h}{ }^{\prime}$ represents the forecast of the total $h$ class load which is based on existing area in year $t$, as the curve a in Figure 4. $L_{t h 1}$ represents the forecast of $h$ class load of existing areas with no history data in year $t$, as the shadow between curve a and curve $b$ in Figure 4. $L_{t h 2}$ represents the forecast of $h$ class load of existing areas with history data in year $t$, as the curve $b$ in Figure 4. $S_{1}$ is the area of $\mathrm{h}$ class existing areas with history data.

In addition, it's so difficult to forecast the load of these existing areas with lots of old buildings to rebuild and the probable changing of site features in short and middle term. Since we cannot control the schedule of rebuilding, it will lead to inaccurate load distribution forecasting. After the rebuilding, it can be forecasted through new areas load forecasting method.

\section{B. New areas load distribution forecasting}

New areas refer to all kinds of residential, commercial, industrial which are developed into pieces, all kinds of new technology development zone and so on, usually are newly designed and developed, basically with no history data, and there is no reference value even with history data. Main thinking shows below:

(1)Determine the classification load development curve of the whole planning area in the vision year. With the development of the city, the emergence of the new areas will make a total area of some kind or certain types of load increases. Gain the total area of classification load from vision year urban land use plans, then combined with the vision classification load saturation density, we can calculate classification load on new planning area in vision year. Then according to similar approach mentioned above, get classification load development curve c.

(2)Estimate the average density of new areas classification load, and forecast the new areas load distribution. According to each category and classification of the load area of the new areas, calculate classification load average density of new areas, calculated as follows:

$$
d_{\text {th,new }}=\frac{\Delta P_{\text {th }}}{S_{\text {th,new }}}
$$

In the formula, first term represents $h$ class load average density of new areas in year $t$; numerator represents $h$ class load of new areas in year $t$, it is from the difference of load value between the load saturation value of the whole planning area and existing area, as the shadow in the top of the figure shown above; denominator represents the area of new areas $h$ class load in year $t$, if the planning time when each new areas appears is uncertain, we can approximately use the total area of increasing new areas from status quo to vision year.

\section{GRID HIERARCHICAL PLANNING MODEL}

High voltage distribution network planning and construction should implement the policy that power has to coordinate with economy, society and environment, strengthen the high voltage distribution network construction and transformation to meet the social and economic development, improve people's living 
standards, consumers' higher and higher requirements for power supply reliability and quality. The basic principle of power network planning is to ensure the power safety and reliability to the load center, so that the power grid construction and operation cost minimum.

\section{A. High voltage distribution network planning model}

Before China electricity power reform, power grid construction lags behind power supply construction. As supporting power supply transmission project, for the purpose to ensure power sent from power plant, power grid becomes the complex of various power plants access system design work, there is a "point to network" phenomenon resulting in weak grid structure. In order to improve the reliability of the power grid, network planning should be "network to point" to ensure safe and stable operation of power system.

Typically grid capacity should meet the peak load requirements, and should have a certain margin. Request in the "Distribution Network Planning and Design Technical Guidelines" shows: $220 \mathrm{kV}$ power substation capacity-load ratio $1.6-1.9,35 \mathrm{kV}-110 \mathrm{kV}$ power substation capacity-load ratio of 1.8-2.1.

The city substation site selection should consider the validation of long-term development of the regional power system planning, considering the network structure, load distribution, urban planning, land acquisition, qualifying corridor, transportation, hydrogeology, environmental impact, seismic intensity and other factors, choose the best solution by comparing the economy of the technical and analyzing economic benefits. High voltage corridor urban planning involved refers to the power line corridor of $110 \mathrm{kV}$ and above. In accordance with the overall urban planning, coordinately arrange the municipal corridors and high-voltage cable channel. There should be no buildings in the scope of the high voltage corridors determined, the location of underground cable channels through the network should be without any other municipal facilities.

High voltage distribution network includes high voltage lines and substations, substation main connection and point-wire connection between various substations determine the grid structure has to meet "N-1" criteria requirements, $35 \mathrm{kV}$ and above substation should have at least double power supply.

\section{B. Medium voltage distribution network planning model}

Main contents of middle voltage substation planning include the division of power range, the radius of power supply, the site selecting of switching stations and distribution transformers. To do these jobs well must combine with urban development planning closely, not only to do load forecasting and load distribution forecasting based on economy development planning and urban land use planning, to divide each high voltage substation supply area, to determine the scope of $10 \mathrm{kV}$ power outlet, the location of the switching station and distribution transformer capacity, but also to cooperate with urban construction planning, to determine the $10 \mathrm{kV}$ line corridor, the location of switching stations and distribution transformers.

To achieve the requirements that medium voltage grid structure is classification and subarea, each voltage level has to be built in ring and open-loop operation. In the distribution network planning, the backbone cable of center region use ring network power supply and the " two minus one" wiring of open-loop operation mode. The $10 \mathrm{kV}$ outlet lines between various substations are linked up by contact switch and disconnected operation, when accidents and maintenance, close the connect switch to ensure continuous power supply to consumer. Under normal circumstances, the maximum load of each feedback wire should be controlled below $50 \%$ of the cable safe ampacity. When $10 \mathrm{kV}$ lines between two substations can't achieve "two minus one" wiring, at least $10 \mathrm{kV}$ lines from different bus bars of the same substation have to achieve "two minus one" wiring. In the subsequent construction and transformation, by increasing the $10 \mathrm{kV}$ feeders, gradually form a "three minus one" ring structure to meet load growth and reliability of power supply requirements.

\section{GRID REGIONAL PLANNING MODEL}

According to "Distribution Network Planning and Design of the Technical Guidelines", divide the power supply area into different regions. Power supply area dividing is primarily based on the administrative level or the load density of planning year, also refer to the degree of the developed economies, the importance of the consumer, consumption level, GDP and other factors.

TABLE 1. POWER SUPPLY AREA TABLE

\begin{tabular}{|c|c|c|c|c|c|c|c|}
\hline \multicolumn{2}{|c|}{ Power supply area } & $\mathrm{A}+$ & A & B & $\mathrm{C}$ & D & E \\
\hline \multirow{4}{*}{$\begin{array}{c}\text { Administrative } \\
\text { level }\end{array}$} & municipality & $\begin{array}{c}\text { City } \\
\text { center } \\
\sigma \geqslant \\
30\end{array}$ & $\left\{\begin{array}{c}\text { Downtown } \\
15 \leqslant \sigma \\
<30\end{array}\right.$ & $\begin{array}{l}\text { Downtown } \\
6 \leqslant 0<15\end{array}$ & $\begin{array}{c}\text { Town } \\
1 \leqslant \sigma<6\end{array}$ & $\begin{array}{c}\text { Countryside } \\
0.1 \leqslant \sigma<1\end{array}$ & - \\
\hline & $\begin{array}{l}\text { Capital city, } \\
\text { Municipalities }\end{array}$ & $\begin{array}{c}\sigma \geqslant \\
30\end{array}$ & $\begin{array}{c}\text { City center } \\
15 \leqslant \sigma \\
<30\end{array}$ & $\begin{array}{l}\text { Downtown } \\
6 \leqslant \sigma<15\end{array}$ & $\begin{array}{c}\text { Town } \\
1 \leqslant \sigma<6\end{array}$ & $\begin{array}{c}\text { Countryside } \\
0.1 \leqslant \sigma<1\end{array}$ & - \\
\hline & \begin{tabular}{|c|} 
Prefecture- \\
level cities \\
(prefectures, \\
leagues)
\end{tabular} & - & $\sigma \geqslant 15$ & $\begin{array}{l}\text { City center } \\
6 \leqslant \sigma<15\end{array}$ & $\begin{array}{c}\text { Downtown } \\
\text { town } \\
1 \leqslant \sigma<6\end{array}$ & $\begin{array}{l}\text { Countryside } \\
0.1 \leqslant \sigma<1\end{array}$ & $\begin{array}{c}\text { Pastoral } \\
\text { area }\end{array}$ \\
\hline & $\begin{array}{c}\text { Counties } \\
\text { (county-level } \\
\text { cities, qi) }\end{array}$ & - & - & $\sigma \geqslant 6$ & $\begin{array}{c}\text { Town } \\
1 \leqslant \sigma<6\end{array}$ & $\begin{array}{l}\text { Countryside } \\
0.1 \leqslant \sigma<1\end{array}$ & $\begin{array}{c}\text { Pastoral } \\
\text { area }\end{array}$ \\
\hline
\end{tabular}

Note1: $\sigma$ is load density of power supply area $\left(\mathrm{MW} / \mathrm{km}^{2}\right)$

Note2: power supply area is more than $5 \mathrm{~km}^{2}$

Note3: When calculating the load density, should deduct $110(66) \mathrm{kV}$ personal load, as well as mountains, desert, desert, water, forests and other ineffective supply area

Power supply area substation capacity configuration should consider load density, space resources, as well as upper and lower grid coordination and overall economy and other factors, to determine the scope of substation power supply and capacity of the sequence of the main power transformer. The same voltage level of the main single transformer capacity standard from the same planning area should not be more than three, the main transformer from the same substation of the same size should be unified. 
TABLE 2. ALL KINDS OF POWER SUPPLY AREA SUBSTATION FINAL CAPACITY CONFIGURATION RECOMMENDED TABLE

\begin{tabular}{c|c|c|c}
\hline Voltage level & $\begin{array}{c}\text { Type of power supply } \\
\text { area }\end{array}$ & number & Single capacity(MVA) \\
\hline \multirow{4}{*}{$110 \mathrm{kV}$} & $\mathrm{A}+, \mathrm{A}$ & $3 \sim 4$ & 63,50 \\
\cline { 2 - 4 } & $\mathrm{B}$ & $2 \sim 3$ & $63,50,40$ \\
\cline { 2 - 4 } & $\mathrm{C}$ & $2 \sim 3$ & $50,40,31.5$ \\
\cline { 2 - 4 } & $\mathrm{D}$ & $2 \sim 3$ & $40,31.5,20$ \\
\cline { 2 - 4 } & $\mathrm{E}$ & $1 \sim 2$ & $20,12.5,6.3$ \\
\hline \multirow{4}{*}{$35 \mathrm{kV}$} & $\mathrm{A}+\mathrm{A}$ & $2 \sim 3$ & $31.5,20$ \\
\cline { 2 - 4 } & $\mathrm{B}$ & $2 \sim 3$ & $31.5,20,10$ \\
\cline { 2 - 4 } & $\mathrm{C}$ & $2 \sim 3$ & $20,10,6.3$ \\
\cline { 2 - 4 } & $\mathrm{D}$ & $1 \sim 3$ & $10,6.3,3.15$ \\
\cline { 2 - 4 } & $\mathrm{E}$ & $1 \sim 2$ & $3.15,2$ \\
\hline
\end{tabular}

Note1:The low voltage side of the main transformer is $10 \mathrm{kV}$

Note2: For determining the power load area, can use small-capacity transformer

Note $3: 31.5 \mathrm{MVA}$ transformer $(35 \mathrm{kV})$ in class $\mathrm{A}+, \mathrm{A}, \mathrm{B}$ region suit the situation that power supply situation from $220 \mathrm{kV}$ substation

The reasonable grid structure is the foundation to meet the power supply reliability, to improve operational flexibility, reduce network losses. High, medium and low voltage distribution network should match each other, keep strong simple and orderly, and support each other in order to achieve the overall distribution network technical and economic optimum.

TABLE $3110 \mathrm{kV} \sim 35 \mathrm{kV}$ GRID TARGET GRID ARCHITECTURE RECOMMENDATION FORM

\begin{tabular}{|c|c|c|c|c|c|c|c|c|}
\hline \multirow[b]{2}{*}{$\begin{array}{c}\text { Voltage } \\
\text { level }\end{array}$} & \multirow{2}{*}{$\begin{array}{c}\text { Type of } \\
\text { power } \\
\text { supply } \\
\text { area }\end{array}$} & \multicolumn{3}{|c|}{ chain } & \multicolumn{2}{|c|}{ Ring } & \multicolumn{2}{|c|}{ radiation } \\
\hline & & $\begin{array}{l}\text { Triple } \\
\text { chain }\end{array}$ & $\begin{array}{c}\text { Double } \\
\text { chain }\end{array}$ & $\begin{array}{l}\text { Single } \\
\text { chain }\end{array}$ & $\begin{array}{c}\text { Double } \\
\text { ring }\end{array}$ & $\begin{array}{c}\text { Single } \\
\text { ring }\end{array}$ & $\begin{array}{c}\text { Double } \\
\text { radiation }\end{array}$ & $\begin{array}{c}\text { Single } \\
\text { radiation }\end{array}$ \\
\hline \multirow{5}{*}{$110(66) \mathrm{kV}$} & $\mathrm{A}+, \mathrm{A}$ & $\sqrt{ }$ & $\sqrt{ }$ & $\sqrt{ }$ & $\sqrt{ }$ & & $\sqrt{ }$ & \\
\hline & $\mathrm{B}$ & $\sqrt{ }$ & $\sqrt{ }$ & $\sqrt{ }$ & $\sqrt{ }$ & & $\sqrt{ }$ & \\
\hline & $\mathrm{C}$ & $\sqrt{ }$ & $\sqrt{ }$ & $\sqrt{ }$ & $\sqrt{ }$ & $\sqrt{ }$ & $\sqrt{ }$ & \\
\hline & $\mathrm{D}$ & & & & & $\sqrt{ }$ & $\sqrt{ }$ & $\sqrt{ }$ \\
\hline & $\mathrm{E}$ & & & & & & & $\sqrt{ }$ \\
\hline \multirow{5}{*}{$35 \mathrm{kV}$} & $\mathrm{A}+, \mathrm{A}$ & $\sqrt{ }$ & $\sqrt{ }$ & $\sqrt{ }$ & $\sqrt{ }$ & & $\sqrt{ }$ & \\
\hline & B & & $\sqrt{ }$ & $\sqrt{ }$ & & $\sqrt{ }$ & $\sqrt{ }$ & \\
\hline & $\mathrm{C}$ & & $\sqrt{ }$ & $\sqrt{ }$ & & $\sqrt{ }$ & $\sqrt{ }$ & \\
\hline & $\mathrm{D}$ & & & & & $\sqrt{ }$ & $\sqrt{ }$ & $\sqrt{ }$ \\
\hline & E & & & & & & & $\sqrt{ }$ \\
\hline
\end{tabular}

Note1: Class A+, A, B power supply areas require high security level, $110 \sim$ $35 \mathrm{kV}$ grid should adopt the chain structure, adopt the double ring network structure when lack of superior power, adopt the double radiation network structure when superior grid is strong and $10 \mathrm{kV}$ has strong inter-station transfer capability.

Note2: Class $\mathrm{C}$ power supply areas require high security level, $110 \sim 35 \mathrm{kV}$ grid should adopt the chain, the ring and also the double radiation structure. Note3: Class D power supply areas $110 \sim 35 \mathrm{kV}$ grid should adopt single radiation structure, and also double radiation or ring structure with condition. Note4: Class E power supply areas $110 \sim 35 \mathrm{kV}$ grid usually adopt single radiation structure
TABLE 4 10kV DISTRIBUTION NETWORK TARGET GRID STRUCTURE RECOMMENDATION FORM

\begin{tabular}{c|c}
\hline $\begin{array}{c}\text { Type of power supply } \\
\text { area }\end{array}$ & Recommended grid structure \\
\hline \multirow{2}{*}{ A,$+ \mathrm{A}$} & Cable lines: double ring, single ring \\
\cline { 2 - 2 } & $\begin{array}{c}\text { Overhead lines: Multi-segment moderately } \\
\text { Contact }\end{array}$ \\
\hline \multirow{2}{*}{$\mathrm{B}$} & $\begin{array}{c}\text { Overhead lines: Multi-segment moderately } \\
\text { Contact }\end{array}$ \\
\cline { 2 - 2 } & Cable lines: single ring \\
\hline \multirow{2}{*}{$\mathrm{C}$} & Overhead lines: Multi-segment moderately \\
& Cable lines: single ring \\
\cline { 2 - 2 } & Overhead lines: Multi-segment moderately \\
D & Contact, radiation \\
\hline E & Overhead lines: radiation \\
\hline
\end{tabular}

\section{EXAMPLE}

\section{A. Examples of time-space load forecasting}

Taking Jinghu District of Wuhu city as an example, to forecast the load of Jinghu District in 2005-2014 years according to the historical data, and verify the accuracy of the model by comparing with the real value.

Jinghu District actual maximum load is 242.5 thousand $\mathrm{kW}$ in the year of 2013 , the total electricity consumption reach 0.849 billion $\mathrm{kWh}$. By time-space load forecasting model, forecast the maximum load is 242.1 thousand $\mathrm{kW}$, the total electricity consumption is 0.823 billion kWh. Jinghu District from 2005 to 2013 contrastive actual and forecasting values of total electricity consumption and maximum load are shown in table 5. As we can see, compared with the actual value, the forecasting values are a slightly lower, but the results are more close to the real value than the conventional forecasting model. The calculation shows that, the temporal and spatial load forecast method to forecast value and the actual value of the error was $0.66 \%$. The regression analysis method error $1.64 \%$, elastic coefficient method error $2.23 \%$, which shows that temporal and spatial load forecasting model is more accurate. 
TABLE5 2005 2013 JINGHU DISTRICT COMPARISON OF PLANNING AREA FORECASTING VALUE AND ACTUAL VALUE UNIT: MILLION kW, ONE HUNDRED MILLION kWh

\begin{tabular}{|c|c|c|c|c|c|c|c|c|c|c|}
\hline & 2005 & 2006 & 2007 & 2008 & 2009 & 2010 & 2011 & 2012 & 2013 & 2014 \\
\hline $\begin{array}{c}\text { The actual } \\
\text { values of total } \\
\text { electricity } \\
\text { consumption }\end{array}$ & 3.77 & 3.86 & 4.26 & 4.99 & 5.73 & 6.45 & 7.29 & 7.71 & 8.49 & 9.11 \\
\hline $\begin{array}{c}\text { Time-space load } \\
\text { forecasting }\end{array}$ & 3.63 & 3.74 & 4.12 & 4.82 & 5.54 & 6.24 & 7.06 & 7.47 & 8.23 & 8.98 \\
\hline $\begin{array}{c}\text { regression } \\
\text { analysis method }\end{array}$ & 3.49 & 3.58 & 3.97 & 4.66 & 5.33 & 6.02 & 6.83 & 7.22 & 7.98 & 8.62 \\
\hline $\begin{array}{c}\text { elastic } \\
\text { coefficient } \\
\text { method }\end{array}$ & 3.37 & 3.46 & 3.80 & 4.46 & 5.13 & 5.80 & 6.62 & 6.98 & 7.72 & 8.43 \\
\hline $\begin{array}{c}\text { The actual } \\
\text { values of the } \\
\text { maximum load }\end{array}$ & 10.76 & 11.04 & 12.17 & 14.25 & 16.36 & 18.43 & 20.83 & 22.03 & 24.2 & 26.03 \\
\hline $\begin{array}{c}\text { Time-space load } \\
\text { forecasting }\end{array}$ & 10.69 & 10.99 & 12.12 & 14.19 & 16.28 & 18.35 & 20.77 & 21.9 & 24.21 & 26.01 \\
\hline $\begin{array}{c}\text { regression } \\
\text { analysis method }\end{array}$ & 10.59 & 10.86 & 12.03 & 14.11 & 16.16 & 18.24 & 20.71 & 21.89 & 24.19 & 25.87 \\
\hline $\begin{array}{c}\text { elastic } \\
\text { coefficient } \\
\text { method }\end{array}$ & 10.52 & 10.81 & 11.86 & 13.95 & 16.02 & 18.12 & 20.69 & 21.8 & 24.14 & 25.84 \\
\hline
\end{tabular}

\section{B. Examples of classification and subarea planning load forecasting}

Taking $110 \mathrm{kV}$ voltage level in Jinghu District of Wuhu city as an example to do the classification and subarea planning. Jinghu District has four $110 \mathrm{kV}$ substation, respectively is the Changjiang Road substation, Jihe substation, Tingtang substation, YangJiamen substation, power grid structure is double radiation and the single chain structure. The $220 \mathrm{kV}$ District of Jinghu old areas have placement difficulties, "four lines and three substations" phenomenon is obvious. $110 \mathrm{kV}$ outlet is difficult, the grid structure is not reasonable, the security risk is big, which affects the power network planning and power grid construction.

In order to solve the network defects, according to the temporal and spatial load forecast and substation capacity balance results, planning to build $110 \mathrm{kV}$ WangJia xiang substation in the year 2018. According to the technical requirements of the grid partition planning model and "Distribution Network Planning and Design of the Technical Guidelines" determine the substation main transformer is $2 \times 50$ MVA, grid structure can use single and double chain, chain, double loop network and double radiation connection form.
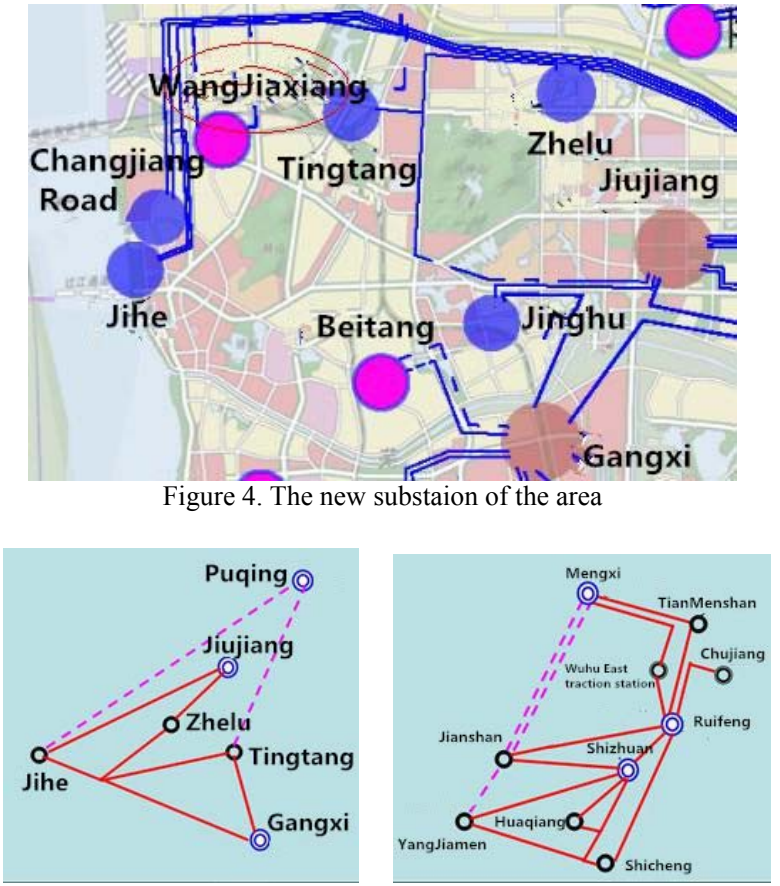

Figure 5. The grid structure optimization of the area

\section{SUMMARY}

"One point two lines" is the planning method of exploring time and space two main lines according to "Urban Power Network Planning and Design Guidelines "and "Distribution Network Planning and Design of the Technical Guidelines". This paper focus on the classification and subarea improved method of spatial load forecasting, propose classification and subarea utility method based on the spatial load forecasting of the vacant area inference. As for the existing classification and subarea, each load area's load density of planning period is determined by the same classification load average density of the entire planning area, correct the deficiency according to the actual load density of load area, divide the load area into existing areas with history data, existing and new areas without history data, based on the theory of vacant area inference, for existing and new areas with and without history data, we use different load forecast ideas, to improve the practicality and effectiveness of the result of distribution network spatial load forecasting.

Classification and subarea means divide the grid into different voltage level, and divide the grid into different regions in the same voltage. Taking "Urban Power Network Planning and Design Guidelines "and "Distribution Network Planning and Design of the Technical Guidelines" as the basis, according to the grid planning in different voltage levels, we can divide it into high-voltage and medium-voltage distribution network planning, planning include load forecasting, substation locating, grid structure planning. According to the administrative level or the load density in planning year, divide the power supply area in the same voltage level into $\mathrm{A}+, \mathrm{A}, \mathrm{B}, \mathrm{C}, \mathrm{D}, \mathrm{E}$ six kinds. And clear the planning technique requirements of different power supply area, include including planning targets, substation capacity configuration, network structure planning. 


\section{REFERENCES}

[1] Nie Hong-zhan, Wang Bi-yuan. Multistage Transmission Network Planning based on Pseudo-dynamic Chaotic Artificial Fish School Algorithm [J]. Advanced Technology of Electrical Engineering and Energy,2011, 30(3) : 75-79.

[2] Edurdo G.Carrano, Luiz A.E.Soares . Electric Distribution Network Multi-objective Design Using a Problem-Specific Genetic Algorithm[J] . IEEE transactions on powerdelivery,2006, $21(2)$ : 995-1005.

[3] Shu Jun, Han Bing, Chen Xuejiao. Spatial Power Network Planning Considering Electric Line Route Optimization[J] , Proceedings of the CSEE, 2014, 34(4) : 570-577.

[4] Xiao Bai, Zhou Chao, Mu Gang. Review and Prospect of the Spatial Load Forecasting Methods Proceedings of the CSEE, 2013, 33(25) : 78-92.

[5] Gong Jiaolong. Transmission Network Expansion Planning based on Ecology Evolutionary Algorithm of Food Chain [D]. Nanchang University, 2010.

[6] Liang Jinzhao, Xia Qing, Wang Dexing. A New Thinking of Group Based Power Grid Planning for Cities With Rapid Growth[J].Power System Technology, 2009, 33(17) : 70-75.

[7] Xie Qin. Research on Spatial Load Forecast for Urban Functional Areas[D]. TianJin University,2012.
[8] Wang Shizhen. Dispatching operation technology [M]. Shenyang: Northeastern University Press, 1997.

[9] Xue Weixing, Cai Yantao, Li Zhiyong. Application of Multi-Layer Decomposition for Spatial Load Forecasting (SLF) in Urban Network Planning, Mechanical \& Electrical Engineering Technology,2014,42(1) : 32-36.

[10] Dalton J G, Garrison D L, Fallon C M. Value Based Reliability Transmission Planning. IEEE Trans on PWRS, 1996, 11(3) : 123-128

[11] Peng Chunhua, Hua Yanwei, Chen Shoukun. Distribution Optimization of DGs in Distribution Networks Based on Hierarchical Partitioning Model[J], Journal of East China Jiaotong University, 2013, 30(2) : 85-89.

[12] Liu Dong, Chen Yunhui, Huang Yuhui.Hierarchical Energy Management and Coordination Control of Active Distribution Network[J], Proceedings of the CSEE, 2014, 34(31) : 5501-5506.

[13] Li Quan. Research on Delamination and Partrition-Area Operation and Its Stability of Hebei Southern Power Grid[J], North China Electric Power University, 2011.

[14] Sun Wansheng, Zhang Hailiang. Electric network voltage-grading and district-diving data managemant system based on GIS[J], Automation and Application, 2011, $12: 49-50$.

[15] Li Yadong. Study on scheme of electric supply for voltage grading and network partition and index algorithm[D], 2007. 